\title{
Mitochondrial trifunctional protein deficiency as a polyneuropathy etiology in childhood
}

\author{
Özlem Ünal Uzun ${ }^{1 \oplus}$, Büşra Çavdarlıㄹ, Selen Karalök ${ }^{3 \oplus}$ \\ ${ }^{1}$ Department of Pediatric Metabolic, Kocaeli University Faculty of Medicine, Kocaeli, ${ }^{2}$ Medical Genetics, Ankara City Hospital, Ankara; \\ ${ }^{3}$ Department of Pediatric Neurology, Akdeniz University Faculty of Medicine, Antalya, Turkey.
}

\begin{abstract}
Background. The mitochondrial trifunctional protein (MTP) is a multienzyme complex of the fatty acid betaoxidation cycle. Mitochondrial trifunctional protein deficiency (MTPD), a rare condition that leads to failure of converting certain fats to energy is characterized by decreased activity of three enzymes in the enzyme complex. Signs and symptoms of MTPD may present during infancy or later in life; those that begin after infancy include hypotonia, muscle pain, rhabdomyolysis, and peripheral neuropathy. We report a Turkish boy diagnosed with MTPD after being investigated for polyneuropathy of unknown origin since infancy.
\end{abstract}

Case. A 5.5-year-old male patient was admitted to our clinic with complaints of weakness in the arms and legs, physical inactivity compared to his peers, fatigue, weakness and, difficulty in climbing stairs since infancy. Electroneuromyography (ENMG) analysis showed moderate symmetric distal sensorimotor and axonal neuropathy. On the background of chronic polyneuropathy, the patient had acute relapsing episodes with progressively worsening severity in the follow-up period until 12.5 years of age. Whole exome sequencing (WES) was performed in the patient and, revealed that the patient had a homozygous c.1390G>A (p.Gly464Ser) pathogenic variant of the $H A D H B$ gene. Although rhabdomyolysis is a well defined accompanying clinical feature of MTPD, it was not present in our patient who only had worsening muscle weakness during attacks.

Conclusion. On the background of chronic polyneuropathy and acute relapsing episodes triggered by fasting or illnesses and rhabdomyolysis physicians should suspect disorders of the fatty acid beta-oxidation cycle.

Key words: polyneuropathy, mitochondrial trifunctional protein deficiency, fatty acid oxidation disorder, $H A D H B$ gene.

The mitochondrial trifunctional protein (MTP) is a multienzyme complex of the fatty acid betaoxidation cycle. It is composed of four alpha and four beta subunits as HADHA and HADHB. The three functions are 2-enoyl coenzyme A (CoA) hydratase (LCEH), long-chain 3-hydroxy acylcoenzyme A dehydrogenase (LCHAD), and 3-ketoacyl CoA thiolase (LCTH). ${ }^{1}$ The HADHA gene encodes the LCEH and LCHAD enzymes and the HADHB gene encodes the LCTH enzyme. The cytogenetic location of both genes is at $2 \mathrm{p} 23.3$. Mitochondrial trifunctional protein deficiency (MTPD) which is a rare condition

凶 Özlem Ünal Uzun

unalozlem@gmail.com

Received 3rd December 2020, revised 27th January 2021, 24th February 2021, accepted 9th March 2021. that leads to failure of converting certain fats to energy is characterized by decreased activity of all three enzymes.

Signs and symptoms of MTPD may begin during infancy or later in life. Clinically, classic MTPD is classified into three main clinical phenotypes including the neonatal onset of a severe, lethal condition resulting in sudden unexplained infant death, the infantile-onset of a hepatic Reye-like syndrome, and the late-adolescent onset of primarily a skeletal myopathy., ${ }^{2,3}$ Symptoms that occur during infancy are various and may include feeding difficulties, hypoglycemia, hypotonia, and liver problems, and these patients may present with heart problems, respiratory insufficiency, coma, and sudden death. Signs and symptoms 
of MTPD that may begin after infancy include hypotonia, muscle pain, rhabdomyolysis, and peripheral neuropathy. Symptoms can be triggered by periods of fasting or by illnesses such as viral infections.

Patients presenting with the prolonged, progressive course of the disease, which has been associated with myopathy, recurrent rhabdomyolysis, and sensorimotor axonal neuropathy tend to survive into adolescence and adulthood. ${ }^{4}$ Here, we report a Turkish patient diagnosed with MTPD who had been under investigation for polyneuropathy of unknown origin since his infancy.

\section{Case Report}

A 5.5-year-old male patient was admitted to our clinic with complaints of weakness in the arms and legs, fatigue, physical inactivity compared to peers and, difficulty in climbing stairs since infancy. His prenatal follow-up was normal and he was born at term with a birth weight of 3160 $\mathrm{g}$ with an uneventful delivery. He was the third child of consanguineous Turkish parents, and his siblings were healthy. He had walked when he was one year old and started using words at 1.5 years old. However, a waddling gate was first noticed at three years of age.

On admission to our center, growth was found to be normal for age. He had mild kyphoscoliosis. Neurological examination revealed absence of deep tendon reflexes and mild waddling gait. Muscle strength was 4/5 in the proximal and distal assessment of all four limbs. Routine investigations including whole blood count, biochemical parameters, Creatine Kinase $(\mathrm{CK})$ level and vitamin $\mathrm{B}_{12}$ level were within normal ranges. An inherited polyneuropathy was suggested in the patient. Metabolic investigations including serum and urine amino acids, serum ammonia level, lactic acid, and pyruvic acid analysis, tandem mass spectrometry, urine organic acid analysis, and very-long-chain fatty acid analysis were normal. Cranial magnetic resonance imaging (MRI) was normal and cardiology and ophthalmology referrals also found no remarkable findings.

In an electroneuromyography (ENMG) analysis performed before admission to our clinic, a sensorimotor neuropathy had been suspected. ENMG analysis was repeated in our center and it showed moderate symmetric distal sensorimotor and axonal neuropathy prominent in the lower extremity. Muscle biopsy showed prominent fiber type grouping which was consistent with secondary neurogenic changes. The patient was investigated for Charcot-MarieTooth disease (CMT), however, the molecular genetic analysis performed for peripheral myelin protein 22 (PMP22) gene mutation was found non-consistent with the diagnosis. Therefore the possibility of mitochondrial disease was suspected in the patient and multivitamin supplements were initiated, which yielded partial benefit.

At 6.5 years of age, he had complaints of walking deterioration and gait disturbance concurrent with an infection. On neurologic examination ataxia was noted in addition to the absence of deep tendon reflexes. Muscle enzymes including CK and lactate dehydrogenase, liver enzymes, renal functions, and electrolytes were checked for rhabdomyolysis and they were within normal ranges. He was investigated for causes of ataxia. Serum alpha-fetoprotein, vitamin $\mathrm{A}$ and vitamin $\mathrm{E}$ levels were normal. Hearing assessment, ophthalmologic evaluation, echocardiography, spinal MRI, and molecular genetic analysis performed to evaluate Friedreich ataxia were also normal. In the follow-up period, the gait disturbance complaint regressed spontaneously. Gait and walking disturbance attacks repeated during infectious illnesses at 9 and 11 years of age. In these two attacks, the severity of symptoms had progressed and he could not even stand and walk, even though muscle enzymes continued to be normal (CK levels were 123 and $140 \mathrm{U} / \mathrm{L}$, respectively). Repeated cranial and spinal MRI studies were unremarkable. ENMG performed at 12 years of age showed chronic severe sensorimotor and axonal neuropathy. Although 
gate disturbance regressed after the course of each attack, his walking became relatively worse at 12.5 years of age and the family declined to use any vitamin supplements.

Whole exome sequencing (WES) was performed in the patient and, revealed that the patient had a homozygous c.1390G>A (p.Gly464Ser) pathogenic variant of the $H A D H B$ gene compatible with MTPD. Parents were detected as heterozygous for the variant. After definitive diagnosis, all routine biochemical and metabolic investigations including serum ammonia level, lactic acid, and pyruvic acid analysis, tandem mass spectrometry, urine organic acid analysis, and ophthalmologic evaluation were performed once again and found normal. Enzyme analysis of the three enzymes forming the MTP could not be performed in the patient due to the fact that these tests can only be performed by very few laboratories related to technical difficulties and financial problems. A low-fat diet with restriction of long-chain fatty acid intake and substitution with medium-chain fatty acids (MCT oil) was commenced. At the last visit, he was 13.5 years old, and he had difficulty standing on his heels. However, his walking and balance were much better under diet and MCT oil treatment.

Informed consent was received from the family.

\section{Evaluation of the Variant:}

The c.1390G $>$ A mutation, which was detected as homozygous in the $H A D H B$ gene in our patient, is a rare variant that has not been detected in healthy population scans previously performed. In the analysis of this variant with in-silico prediction programs, it was evaluated as a disease-causing variant. This variant, which has not been reported in the literature and online databases, is classified as a "variant of unknown significance" according to the American College of Medical Genetics and Genomics variant classification guidelines. ${ }^{5}$ However, since our patient's clinical findings and clinical progression were compatible with MTPD, this variant was thought to be responsible for the patient's clinic.
The variant corresponds to the first nucleotide of the exon 16 in the genomic sequence. At the translation level, this variant could create a new possible splice site. The analyses according to "Human Splicing Finder", the c.1390G>A variant at the beginning of the coding region is estimated to create a new acceptor site (New site: +58.9). ${ }^{6}$ If a new acceptor site has occurred, the structure of the protein may have changed.

The evaluation at the level of amino acid exchange; glycine at the position 464 , is the first amino acid of the beta-strand of the protein. The variant is replaced by the $C$ terminal of the Thiolase domain (Fig. 1a). The newly formed Serin amino acid has an extra-OH group that may cause the formation of a new hydrogen bond (Fig. 1b, c). ${ }^{7}$ A new hydrogen bond may decrease the stability of the protein by changing the interatomic interactions, atomic fluctuation, and local flexibility (Fig. 1d,e,f). ${ }^{8}$

Polyphen-2 score: 1

PROVEAN: -5.403 (cutoff:-2,5)-deleterious

\section{Discussion}

Inborn errors of metabolism (IEMs) can present at any age from childhood to adulthood and may affect the peripheral nervous system, usually as part of a diffuse neurological or systemic clinical picture. Inherited metabolic neuropathies comprise a clinically, biochemically, and genetically heterogeneous group of diseases. Rarely, neuropathy can be the unique initial sign of an IEM. ${ }^{9}$ Rare causes of metabolic neuropathy include fatty acid betaoxidation disorders, mitochondrial disorders, porphyria, disorders of the lipid or glycolipid metabolism (eg. Refsum disease, Fabry disease, abetalipoproteinemia, Tangier disease). MTPD is a fatty acid beta-oxidation cycle disorder, and is characterized by a wide clinical spectrum ranging from severe neonatal manifestations including cardiomyopathy, hypoglycemia, metabolic acidosis, skeletal myopathy and neuropathy, liver disease, and death to a mild phenotype with peripheral polyneuropathy, 


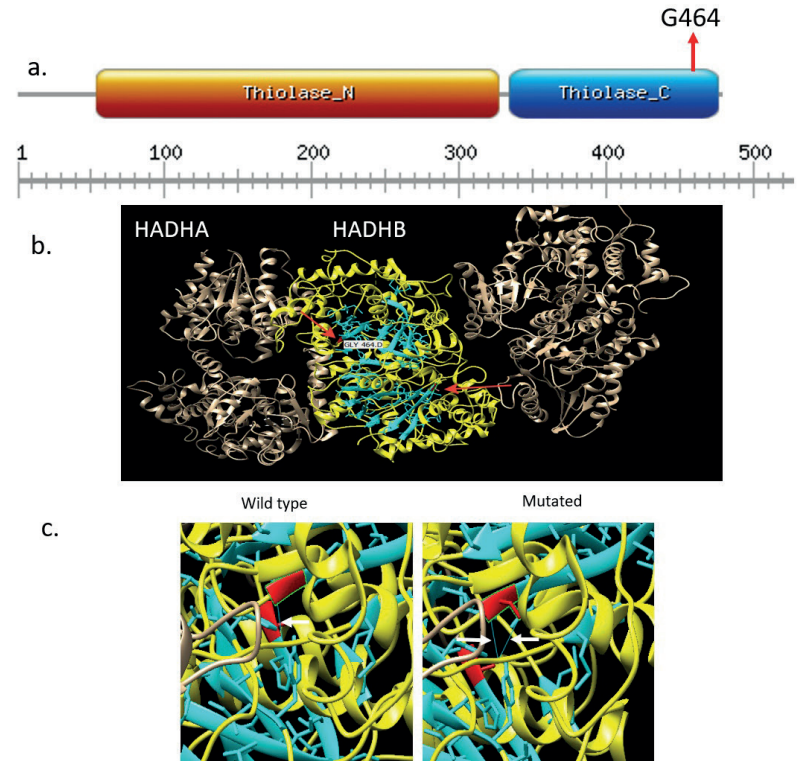

d.

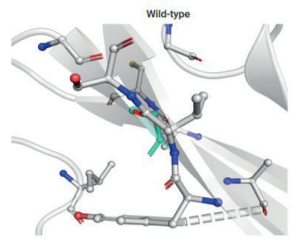

e.

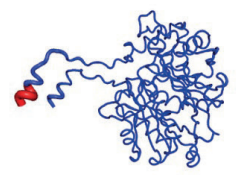

f.

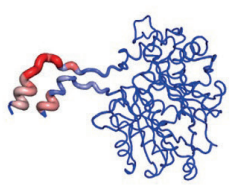

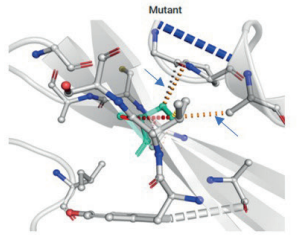
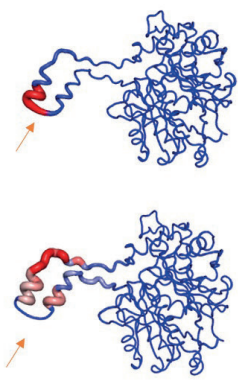

Fig. 1a. Protein domains of HADHB gene; b. 3D structure of Mithocontrial trifunctional protein; $\mathbf{c}$. it is predicted that the mutated protein has an extra $\mathrm{H}$ bond $\mathbf{d}$. Blue bars indicate possible new interatomic interactions, e. $\mathrm{H}$ bonds may provide change in the amplitude of absolute atomic action by decreasing atomic fluctuation, $\mathbf{f}$. and local flexibility.

episodic rhabdomyolysis and pigmentary retinopathy. ${ }^{10}$

The most common motor symptom of peripheral neuropathy is weakness. It may present as clumsiness, difficulty in climbing stairs, or impaired fine motor skills. These motor symptoms were present in our patient since infancy. Ataxia is another motor symptom associated with neuropathy. Ataxia was a prominent symptom in our patient only during the course of deteriorating attacks that developed in association with an infection. Sensory symptoms, of which none were seen in our patient may include numbness, paresthesia, pain, or burning sensations.

Fu et al. ${ }^{11}$ reported an 8 -year-old girl with lower limb weakness since birth who was ultimately diagnosed with MTPD. Blood acylcarnitine analysis revealed slightly increased long-chain 3-OH-acylcarnitine levels; EMG suggested peripheral nerve injury; muscle biopsy confirmed a neurogenic lesion in muscle fibers, as shown by EMG. Analysis of the $H A D H B$ gene identified a homozygous missense mutation c.739C > T (p.R247C). They stated that neonatal- onset, as seen in their patient, has not been reported for the neuromyopathic phenotype of mitochondrial trifunctional protein deficiency. ${ }^{9}$ Clinical findings of our patient were similar to their patient, but repeated acylcarnitine analysis including in deterioration attacks were normal in our patient.

Naiki et al. ${ }^{12}$ reported peripheral polyneuropathy, rhabdomyolysis, and infantileonset hypoparathyroidism in two siblings with $H A D H B$ gene mutations. Recently, van Vliet et al. $^{13}$ reported a 20-year-old woman who had axonal motor-sensory polyneuropathy of unknown origin since childhood. She presented with progressive dyspnea, and increased muscle weakness which had been preceded by an infectious disease. Laboratory testing showed rhabdomyolysis and hypocalcemia with low parathyroid levels. Sequence analysis of the $H A D H B$ gene showed two heterozygous variants. The authors emphasized the importance of performing metabolic screening when patients are most symptomatic, since normal results could be inconclusive in the absence of metabolic stress. ${ }^{11}$ Serum calcium, 
phosphorus, and magnesium levels were normal in our patient, and rhabdomyolysis was not seen, however, he experienced deterioration attacks concurrent with infectious illnesses.

Yamamoto et al. ${ }^{14}$ reported a 45 -year-old man presenting with slowly progressive muscle weakness and sensory disturbances in his lower limbs and multiple episodes of exerciseinduced severe muscle fatigue and brown urine during his childhood, which had disappeared by age 20. A nerve conduction study showed peripheral axonal neuropathy. Similar to our patient, they considered CMT disease, however, exome sequencing failed to identify a mutation in the known genes associated with CMT. Then, he recurrently developed severe rhabdomyolysis that required hospitalization. They re-examined exome sequencing and found a mutation in $H A D H B$. This case of adultdiagnosed MTP deficiency was characterized by slowly progressive peripheral neuropathy masquerading CMT in addition to muscular symptoms. ${ }^{14}$

No pathogenic variant has been identified in exon 16 of the HADHB gene so far, which may be pathologic for the disease. However, it has been reported that mutations detected in the last exons of the gene may be presented with late-onset and milder clinical findings. ${ }^{15}$

The lack of enzyme level measurement is a limitation of this report. However using online modeling and prediction programs, we have been able to predict that the mutation identified herein can destabilize the structure of the protein. Although acylcarnitine levels were repeatedly normal in our patient, clinical findings may be the result of conformational changes in the protein by the Gly464Ser homozygous variant that may lead to a less functional protein. Nevertheless, functional studies are required to clearly understand the effect of the variants that have not any clinical significance yet.

In conclusion, it is evident that the differential diagnosis of isolated polyneuropathy in childhood may be challenging. Most peripheral nerve disorders in children are hereditary. Characterization of a peripheral nerve disorder involves a careful neurologic examination, family history, electrodiagnostic studies. Although rhabdomyolysis is a well-defined accompanying clinical feature of MTPD, muscle weakness that is getting worse in attacks in the absence of rhabdomyolysis was an interesting aspect of our patient. On the background of chronic polyneuropathy, acute relapsing episodes triggered by fasting or illnesses and rhabdomyolysis should suggest the fatty acid beta-oxidation cycle.

\section{Acknowledgement}

Molecular graphics images were produced using the UCSF Chimera package from the Resource for Biocomputing, Visualization, and Informatics at the University of California, San Francisco (supported by NIH P41 RR-01081).

\section{Author contribution}

OU and SK collected the data; OU wrote the manuscript; BC performed genetic analysis.

\section{Conflict of interest}

Authors declare no conflict of interest.

\section{REFERENCES}

1. Morris AAM, Spiekerkoetter U. Disorders of mitochondrial fatty acid oxidation and riboflavin metabolism. In: Saudubray JM, Baumgartner MR, Walter J (eds). Inborn Metabolic Diseases: Diagnosis and Treatment (6th ed). Berlin: Springer 2016: 204205. https://doi.org/10.1007/978-3-662-49771-5_12

2. Spiekerkoetter U, Sun B, Khuchua Z, Bennett MJ, Strauss AW. Molecular and phenotypic heterogeneity in mitochondrial trifunctional protein deficiency due to beta-subunit mutations. Hum Mutat 2003; 21 : 598-607. https://doi.org/10.1002/humu.10211

3. Marucci G, Cianferotti L, Beck-Pecoz P, et al. Rare diseases in clinical endocrinology: a taxonomic classification system. J Endocrinol Invest 2015; 38: 193-259. https://doi.org/10.1007/s40618-014-0202-6 
4. den Boer ME, Dionisi-Vici C, Chakrapani A, van Thuijl AO, Wanders RJ, Wijburg FA. Mitochondrial trifunctional protein deficiency: a severe fatty acid oxidation disorder with cardiac and neurologic involvement. J Pediatr 2003; 142: 684-689. https://doi. org/10.1067/mpd.2003.231

5. Richards S, Aziz N, Bale S, et al; ACMG Laboratory Quality Assurance Committee. Standards and guidelines for the interpretation of sequence variants: a joint consensus recommendation of the American College of Medical Genetics and Genomics and the Association for Molecular Pathology. Genet Med 2015; 17: 405-424. https://doi.org/10.1038/ $\operatorname{gim} .2015 .30$

6. Desmet FO, Hamroun D, Lalande M, Collod-Béroud G, Claustres M, Béroud C. Human Splicing Finder: an online bioinformatics tool to predict splicing signals. Nucleic Acids Res 2009; 37: e67. https://doi. org/10.1093/nar/gkp215

7. Pettersen EF, Goddard TD, Huang CC, et al. UCSF Chimera--a visualization system for exploratory research and analysis. J Comput Chem 2004; 25: 1605-1612. https://doi.org/10.1002/jcc.20084

8. Rodrigues $\mathrm{CH}$, Pires DE, Ascher DB. DynaMut: predicting the impact of mutations on protein conformation, flexibility and stability. Nucleic Acids Res 2018; 46: W350-W355. https://doi.org/10.1093/ nar/gky300

9. Sedel F, Barnerias C, Dubourg O, Desguerres I, Lyon-Caen O, Saudubray JM. Peripheral neuropathy and inborn errors of metabolism in adults. J Inherit Metab Dis 2007; 30: 642-653. https://doi.org/10.1007/ s10545-007-0684-x
10. Carrier Genetic Test Igenomix CGT. Diseases list. Available at: https://cgt.igenomix.ae/diseases-list/

11. Fu X, Zheng F, Zhang Y, et al. Mitochondrial trifunctional protein deficiency due to HADHB gene mutation in a Chinese family. Mol Genet Metab Rep 2015; 5: 80-84. https://doi.org/10.1016/j. ymgmr.2015.10.015

12. Naiki M, Ochi N, Kato YS, et al. Mutations in HADHB, which encodes the $\beta$-subunit of mitochondrial trifunctional protein, cause infantile onset hypoparathyroidism and peripheral polyneuropathy. Am J Med Genet A 2014; 164: 11801187. https://doi.org/10.1002/ajmg.a.36434

13. van Vliet $P$, Berden $A E$, van Schie $M K M$, et al. Peripheral neuropathy, episodic rhabdomyolysis, and hypoparathyroidism in a patient with mitochondrial trifunctional protein deficiency JIMD Rep 2018; 38: 101-105. https://doi. org/10.1007/8904_2017_37

14. Yamamoto Y, Matsui N, Hiramatsu Y, et al. Mitochondrial trifunctional protein deficiency: an adult patient with similar progress to CharcotMarie-Tooth disease. Rinsho Shinkeigaku 2017; 57: 82-87. https://doi.org/10.5692/clinicalneurol.cn000976

15. Purevsuren J, Fukao T, Hasegawa Y, et al. Clinical and molecular aspects of Japanese patients with mitochondrial trifunctional protein deficiency. Mol Genet Metab 2009; 98: 372-377. https://doi. org/10.1016/j.ymgme.2009.07.011 